\title{
Low Electromagnetic Noise Squirrel Cage Motor Analysis and Optimization of Slot Coordination
}

\author{
Changqi Liu \\ Shanghai Dian Ji University \\ Min Hang, District, Shanghai 200240, China; \\ 542368586@qq.com \\ Yufa Xu \\ Shanghai Dian Ji University \\ Min Hang , District, Shanghai 200240, China; \\ xuyf@sdju.eu.cn
}

\author{
Gong $\mathrm{Pu}$ \\ ABB Shanghai Motor Co., Ltd, \\ Min Hang District Shanghai 201613, China; \\ pugong@163.com \\ Jinquan Feng \\ ABB Shanghai Motor Co., Ltd, \\ Min Hang District Shanghai 201613, China; \\ Hanson-Jinquan.feng@cn.abb.com
}

\begin{abstract}
The electromagnetic noise of motor is mainly caused by the main magnetic fluxing into the air gap along the radial direction, generating radial force in the tooth of stator and rotor, which causes electromagnetic vibration and noise. The paper analyzes the theory of the production principle of electromagnetic force by selecting different slot combination to verify the influence on the electromagnetic wave, further conducts through the finite element analysis and modal analysis to prove the validity of the theory, and finally to prove that the choice of slot combination has an important influence on the noise of squirrel cage induction motor through noise test tank.
\end{abstract}

Keywords-Slot Combination; Electromagnetic Force; Finite Element Analysis; Modal Analysis; Noise Test

\section{INTRODUCTION}

At present, harmonic magnetic field of stator and rotor of the squirrel cage induction motor will interact with each other which will produce radial electromagnetic wave changing with time and space when it is electricity in engineering. Radial electromagnetic wave will cause vibration of stator, which will cause the noise. This is the main reason for the motor to generate electromagnetic noise. Intrinsic mode of motor stator structure and radial electromagnetic wave on the surface of the stator core are two major factors to determine the size of induction motor electromagnetic noise .At the same time the selection of slot coordination of the motor is mainly based on experience, and sometime the electromagnetic noise will exceeds standard. Therefore, it is necessary to research the slot coordination selection method of squirrel-cage motor. At the same time, the appropriate or not of slot coordination, not only should consider the order, a mplitude and size of the wave force, must also consider the frequency of force wave and the natural frequency of the stator size, to avoid resonance.
The generation of electromagnetic noise have a close relationship with the radial electromagnetic force wave and the existing mechanical mode, these two aspects should be in consider when choosing low noise slot coordination. Literature 2 studied effect on noise of 8 poles with 28 slots, 8 poles with 36 slots and 8 poles with 48 slots, when this three pole with slot coordination. Literature 3 only studied the electromagnetic wave through groove cooperates select to limit the influence of noise, but did not consider the modal effect. Literatures 4 studied the mode of traction drive motor, but had no contrast on slots with different effects on noise.

Based on the squirrel cage induction motor as an example for slot coordination which is commonly used in engineering analysis the influence of the electromagnetic wave in theoretically in this paper, And in this paper a calculation is made for a company to provide prototype theory analysis and simulation, and through the prototype experiments validate the importance of selecting slot coordination and shorten the design cycle, to reduce the electromagnetic noise in the primary stage fullest.

\section{MAGNETIC WAVE ANALYSIS OF SQUIRREL CAGE INDUCTION MOTOR}

Magnetic field of motor air-gap is used for stator core inner. The value and distribution of the radial electromagnetic force of the surface per unit area which is in accordance with the law of maxwell, is proportional to the square of the magnetic flux density, and it can be determined by Equation (1)

$$
p_{n}(\theta, t)=\frac{b^{2}(\theta, t)}{2 \mu_{0}}
$$


the $\mu_{0}=4 \pi \times 10^{-7} \mathrm{~h} / \mathrm{m}$ in the Equation, $b(\theta, t)$ is for the air gap flux density

When ignore the saturated air gap flux density researchers get Equation (2)

$$
\begin{aligned}
& b(\theta, t)=f(\theta, t) \lambda(\theta, t) \\
& \lambda(\theta, t) \text { is for the air gap permeancein the }
\end{aligned}
$$

Equation(2); $f(\theta, t)$ is f or the air gap magnetic potential. The Equation which means the synthesis of magnetic potential and the product of the air gap permeance when the motor running5.Therefore, researchers get Equation (2) into (1), at the same time omit the higher order number, amplitude of the smaller force component, and omit constant weight at the same time, because they are the basic won't produce vibration noise. Therefore, researchers can get next Equation (3)

$$
p_{n}(\theta, t)=\frac{1}{2 \mu_{0}}\left\{\begin{array}{l}
\frac{B_{1}^{2}}{2} \operatorname{COS}\left(2 p \theta-2 \omega_{1} t-2 \varphi_{0 r}\right)+ \\
\sum_{v_{z} u_{z}} B_{v z} B_{u z} \cos \left[\begin{array}{l}
(u \pm v) \theta-\left(\omega_{u} \pm \omega_{1}\right) t \\
-\left(\varphi_{u r} \pm \varphi_{v r}\right)
\end{array}\right]
\end{array}\right\}
$$

The first part in the equation is (the two times the power frequency) vibration, which is one of the main component of the vibration of motor. Due to many reason has very low frequency of the motor, at the same time, two times the power frequency of vibration is produced by fundamental of air-gap magnetic field, so it is ineviTable, but for the squirrel cage induction motor, the frequency vibration of the size effect on the stator vibration amplitude value is bigger, but due to the effect of attenuation of the ear, the influence of the noise is not big, generally not be considered. The second part is due to the stator and rotor tooth harmonic wave, the force created by the interaction of them generally is the main component of electromagnetic noise, the general order number of force wave is small, and the amplitude is large, and frequency distribution in the human ear sensitive area, therefore, choose the appropriate slot cooperates to suppress the force wave is one of the important measures to reduce the electromagnetic noise6.Electro magnetic wave of air gap in Table1.

TABLE I. ELECTROMAGNETIC WAVE OFAIR GAP

\begin{tabular}{|c|c|c|}
\hline $\begin{array}{c}\text { Categor } \\
\mathrm{y}\end{array}$ & Order & Frequency \\
\hline $\begin{array}{c}\text { Fundamental } \\
\text { force }\end{array}$ & $2 p$ & $2 \omega$ \\
\hline $\begin{array}{c}\text { Harmonic } \\
\text { Force }\end{array}$ & $\mu_{z}+v_{z}$ & $\begin{array}{c}\left(\omega_{u}+\omega_{1}\right) \\
=k_{2} \frac{Z_{2}}{p}(1-S) \omega_{1}+2 \omega_{1}\end{array}$ \\
\cline { 2 - 3 } & $\mu_{z}-v_{z}$ & $\left(\omega_{u}-\omega_{1}\right)=k_{2} \frac{Z_{2}}{p}(1-S) \omega_{1}$ \\
\hline
\end{tabular}

In the Table

$v_{z}=\left(6 k_{1}+1\right) p, u_{z}=k_{2} Z_{2}+P, k 1, k 2= \pm 1, \pm 2, \cdots$
Table 1 illustrate that main frequency of the electromagnetic wave in the air gap under the available Equation can be said:

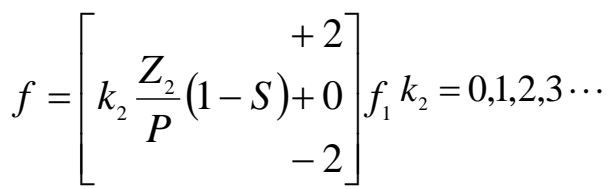

The $n$ means the order of force wave.

Known from the analysis of the above force wave number associated with slot coordination 8, this paper selects slot coordination which is $36 / 20,36 / 22$ and $36 / 24$, and then makes the comparative analysis. From the analysis of the above, researchers list possible order wave of force (less than 6 times) when the prototype in these three slot coordination, as shown in Table 2 to Table 4.

TABLE II

ELECTROMAGNETIC WAVE OF 36/20

\begin{tabular}{|c|l|l|l|l|l|l|}
\hline \multirow{2}{*}{$\begin{array}{c}\text { Roto } \\
r\end{array}$} & \multicolumn{7}{|c|}{ Stator } \\
\cline { 2 - 7 } & -17 & 19 & 23 & 25 & -29 & 31 \\
\hline-23 & 6 & -4 & 0 & 2 & -6 & \\
\hline 25 & & -6 & 2 & 0 & -4 & 6 \\
\hline-47 & & & & & & \\
\hline 49 & & & & & & \\
\hline
\end{tabular}

TABLE III.

ELECTROMAGNETIC WAVE OF 36/22

\begin{tabular}{|l|l|l|l|l|l|l|}
\hline \multirow{2}{*}{ Rotor } & \multicolumn{7}{|c|}{ Stator } \\
\cline { 2 - 7 } & -17 & 19 & -23 & 25 & -35 & 37 \\
\hline-19 & 2 & 0 & -4 & & & \\
\hline 21 & 4 & -2 & -2 & 4 & & \\
\hline-39 & & & & & 4 & -2 \\
\hline 41 & & & & & 6 & -4 \\
\hline
\end{tabular}

TABLE IV.

ELECTROMAGNETIC WAVE OF 36/24

\begin{tabular}{|l|l|l|l|l|l|l|}
\hline \multirow{2}{*}{ Rotor } & \multicolumn{7}{|c|}{ Stator } \\
\cline { 2 - 7 } & -17 & 19 & -23 & 25 & -29 & 37 \\
\hline-21 & 4 & -2 & -2 & 4 & & \\
\hline 23 & 6 & & 0 & 2 & -6 & \\
\hline-43 & & & & & & -6 \\
\hline 45 & & & & & & \\
\hline
\end{tabular}

TABLE V.

ELECTROMAGNETIC WAVE FREQUENCY

\begin{tabular}{|l|l|l|l|}
\hline \multirow{2}{*}{$\begin{array}{l}\text { Number } \\
\text { of force }\end{array}$} & \multicolumn{3}{|c|}{ Slot cooperates } \\
\cline { 2 - 4 } & $36 / 20$ & $36 / 22$ & $36 / 24$ \\
\hline 2 & $1000 / 1900$ & 1000 & 1300 \\
\hline 4 & 1100 & 1100 & 1100 \\
\hline
\end{tabular}

TABLE VI.

MECHANICS PARAMETERS OF MODAL MATERIAL

\begin{tabular}{|c|c|c|c|}
\hline Property & Stator & Stator Shell & Winding \\
\hline $\begin{array}{c}\text { Density } \\
\mathrm{kg} / \mathrm{m} \mathrm{3}\end{array}$ & 7750 & 7100 & 5942 \\
\hline $\begin{array}{c}\text { Elasticity } \\
\text { Modulus/ } \\
\text { Gpa }\end{array}$ & 220 & 78 & 95 \\
\hline $\begin{array}{c}\text { Poisson's } \\
\text { Ratio }\end{array}$ & 0.29 & 0.26 & 0.3 \\
\hline
\end{tabular}

By fin ite element method to calculate the tooth density distribution of the electromagnetic when motor is in the rated speed, then researchers obtained Fourier 
decomposition, and further verifies the correctness of the above calculation which is shown in Fig. 1.

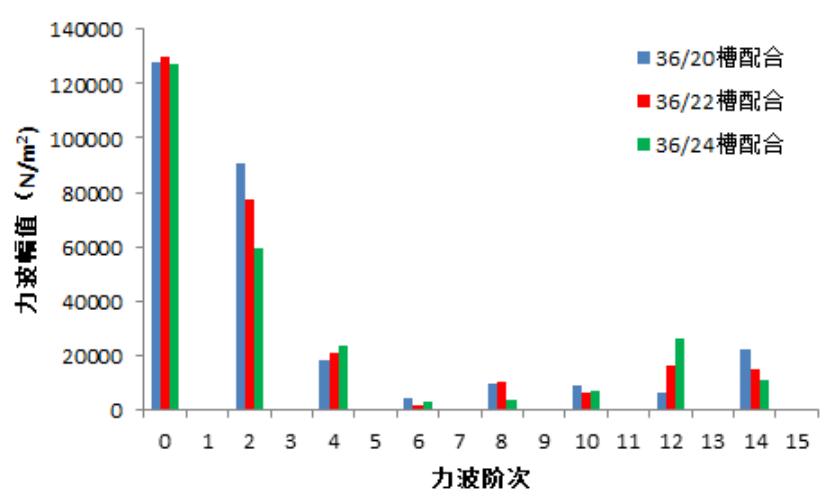

Figure 1. Order of radial force

The Finite element analysis results show that the amplitude value of zero order force is basically the same when the motor in the three tank. Through theoretical analys is researchers know, it won't produce vibration noise. Usually the amplitude of dynamic deformation when the core vibration is inversely proportional with order of radial electromagnetic force, the lower order of radial electromagnetic force, the greater the distance between two adjacent nodes of motor deformation, the greater the radial deformation. Therefore, researchers only study the vibration noise caused by force of the low-order, wave, ignore the existence of higher order wave force, the amplitude of the order is 6 or above of the electro magnetic wave is not a main source of the vibration noise in this paper, when the slot coordination is $36 /, 20$, there is a first order tooth harmonic will produce the electromagnetic force. Therefore, the amp litude of 2 order electro magnetic force is higher, at the same time when slot coordination is $36 /, 22$; the amplitude of 2 order force wave is higher than $36 / 24$ slot coordination.

\section{MODAL ANALYSIS}

This paper researchers use the mechanical modal analysis module of ANSYS, a finite element analysis software the general analysis process including model establishment, set the material properties, and then to evaluate and post-processing, etc. At the same time due to the motor stator core is complex, the stator core is consist by silicon steel sheet together in the actual manufacturing. The traditional analytic method to calculate the modal is simplified by structure, put a lot of connection part as an organic whole, in fact the simplified has been far away from the actual situation, the calculation results is not very accurate. This paper simulated the actual structure of the stator core connecting method, considering the overlying coefficient and casing under the precondition of ignoring rotor and bearing, etc., the core and the casing and the windings are analyzed and calculated by equivalent material parameters as shown in Table 6 by adopting the shown in Fig. 2, the finite ele ment analys is and calculation with the stator iron core to coincide with the actual modal vibration mode.

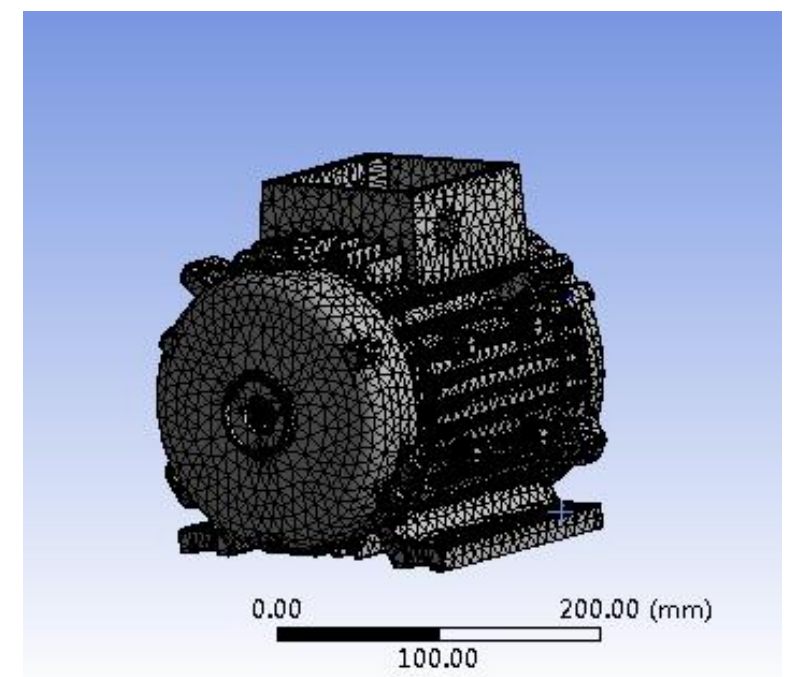

Figure 2. Finite element mesh dissection of stator structure

Known from the analys is of above, the low-order radial electromagnetic force for example when the order is 2 and 4, the electromagnetic force is prominent, and the amplitude of high order electromagnetic force is smaller, the impact on the vibration noise of the motor can be ignored. this paper extract the second order and fourth order modal results of structure of stator and stator core as shown in Fig. 3 to Fig. 6.

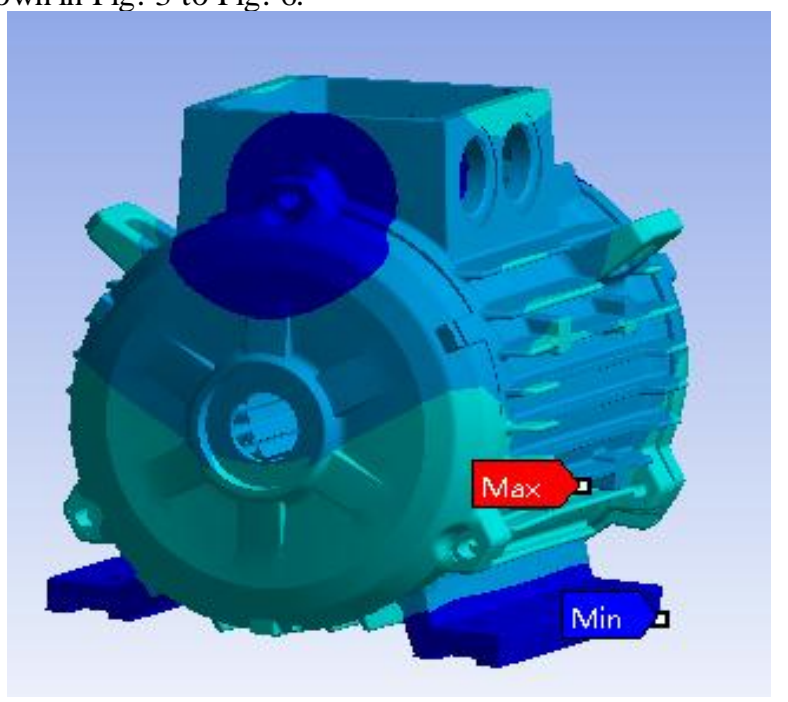

Figure 3. The second order formation of stator structure 


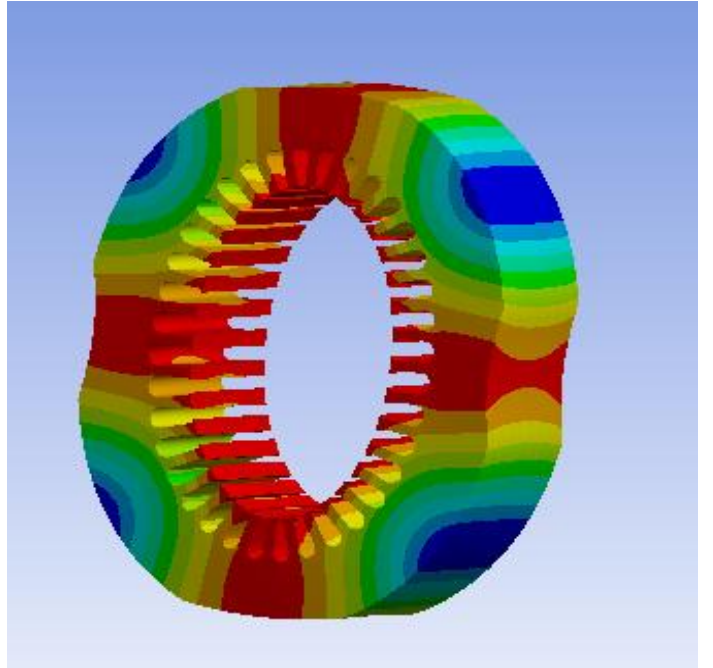

Figure 4. The second order formation of stator

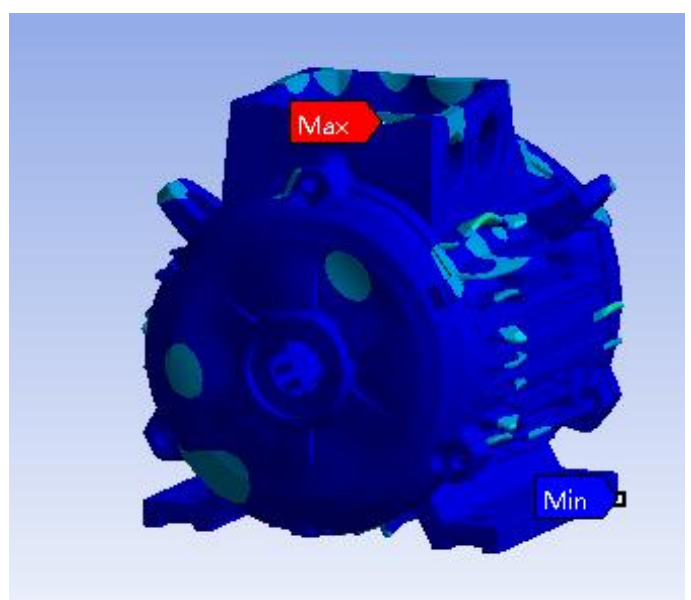

Figure 5. The fourth order formation of stator structure

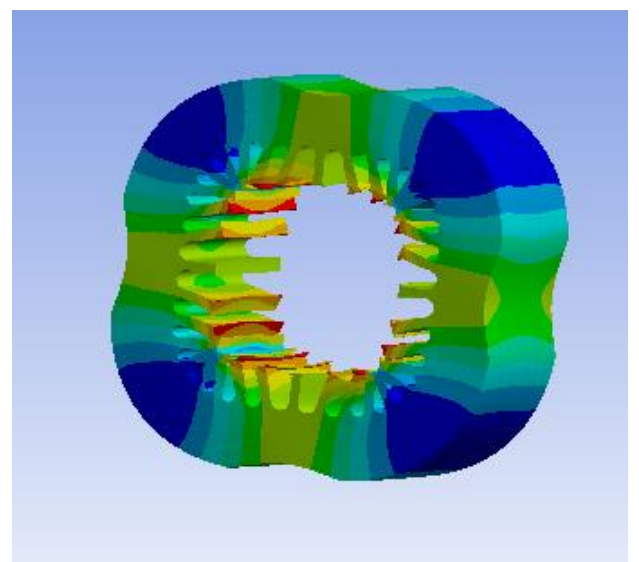

Figure 6. The fourth order formation of stator

TABLE VII. MAIN VIBRATION MODE

\begin{tabular}{|c|c|}
\hline Order & Frequency/Hz \\
\hline 2 & 2022 \\
\hline 4 & 3034 \\
\hline
\end{tabular}

If the stator resonance caused by the force of a wave to produce high electromagnetic noise, it requires two conditions: The first is the order of force wave and to order of stator modal weeks is the same; The second is the frequency of force wave and natural frequency of circumferential order are close .Therefore, compare the electromagnetic force, and the modal analysis results as shown in Table 7 shows 36/20 slot coordination with its order 2 force wave frequency and 2 order formation close to the natural frequency of the motor, so it is easy to produce large noise caused by the resonance, the slot coordination is unreasonable.

\section{NOISE TEST}

In this paper, researchers adept the experimental method to validate the result of the above analysis, according to three kinds of motor slot coordination which is put in the half the mute indoor test platform, through the sound device, at the same time make the motor run in 380 v / $50 \mathrm{~Hz}$ working status, by adopting instrument BK noise meter to test point pressure 9,10 .

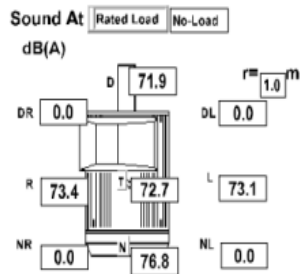

\begin{tabular}{ll|l} 
Background Noise & 0.0 & 40.0 \\
\hline & 00 & 00 \\
\hline
\end{tabular} \begin{tabular}{l|l|l|}
\hline Sound Pressure Level a andigtos & 0.0 & 74.0 \\
\hline
\end{tabular} \begin{tabular}{cccc}
\hline Environmental Correction & 0.0 & 0.0 \\
\cline { 2 - 3 } & & &
\end{tabular}

Figure 7. Noise testing method for rotor slot 22

TABLE VIII. TEST DATA NOISE OF PROTOTYPE

\begin{tabular}{|l|l|l|l|}
\hline Rotor slot & $\begin{array}{l}\text { 20 slots } \\
\mathrm{dB}(\mathrm{A})\end{array}$ & $\begin{array}{l}\text { 22slots } \\
\mathrm{dB}(\mathrm{A})\end{array}$ & $\begin{array}{l}\text { 24slots } \\
\mathrm{dB}(\mathrm{A})\end{array}$ \\
\hline Test point 1 & 71.9 & 69.4 & 66.8 \\
\hline Test point 2 & 73.4 & 73.4 & 69.8 \\
\hline Test point 3 & 72.7 & 73.0 & 69.6 \\
\hline Test point 4 & 73.1 & 73.0 & 69.4 \\
\hline Test point 5 & 76.8 & 74.0 & 73.7 \\
\hline
\end{tabular}

The experiments with different slot coordination are as shown in Table 8 researchers can be, found, the level of motor noise change is bigger, when slot coordination is $36 / 20$. And the noise sound pressure average level is 74.0. When slot coordination is $36 /, 22$, motor noise sound pressure average level is 72.5 . When slot coordination is $36 / 24$, motor noise sound pressure average level is 71 . Therefore, when slot coordination is $36 /, 24$, the motor noise will be the minimum value.

\section{CONCLUSIONS}

In this paper, through theoretical analysis influence of different slot with the electric magnetic force wave and through the finite element method verify the rationality of theoretical analysis. Further to ignore such as rotor modal affected parts of motor stator machine on the basis of modal analysis, researchers forecast the 36/20 and 36/22 slot motor may exist large noise, and through the experiment to prove analysis effectively on the above. In this paper, researchers can get the following conclusions through analysis : the motor noise impact of slot coordination in the design process of the low noise motor is bigger, and researchers should choose reasonable motor 
slot coordination as far as possible to ensure low amplitude of electromagnetic force and difference of its frequency with the inherent vibration mode of the motor is bigger.

\section{ACKNOWLEDGMENT}

This paper is supported by the Natural Science Foundation of Shanghai (No.11ZR1413900), Shanghai Educational Committee Leading Academic Discipline Project (No. J51901), Shanghai Educational Committee key scientific research project (No.09ZZ211), Shanghai Economic and Information Technology Committee (No.13X1-37), Science and Technology Commission of Min Hang Municipality (No.2013MH180), the Key Discipline Project of Shanghai Dian-jiUniversity(No.09XKJ01). Especially, researchers are highly grateful to the referees and Editor-in-Chief for their valuable comments.

\section{REFERENCES}

[1] Yongiiao Chen, Ziqiang Zhu, Shangcheng Ying. Control and analysis of motor noise $[\mathrm{M}]$. Hanghou: zhejiang university press 1987.
[2] Yueping Mo, Minqiang Hu, Zhike Xu. Ultrasonic motor vibration modal finite element analysis [J]. Proceedings of the csee, 2002, 22:92.96.

[3] Xun Wang, Qiu Arui. Cage Equation asynchronous motor of radial electromagnetic wave finite element calculation [J]. Journal of electrotechnics, 2012, 27:109117.

[4] Ying Dai, shumei cui, liwei song. finite element modal analysis of vehicle motor [J]. Proceedings of the CSEE, 2011, Vol.32 No.104.

[5] Yang, s. j. low noise motor [M]. Beijing: science press, 1985.

[6] Chen Shikun. Motor design [M]. Beijing: mechanical industry publishing house, 2000.

[7] ZhixinHuang,Chengzhu Liu[M].ANSYSWorkbench14.0 manual. Beijing:People's posts and telecommunications publishing, 2014

[8] Ying Dai, Shumei Cui, Qianfan Zhang. Analysis on Electromagnetic Vibration/Noise of Induction Motors for EV Drives[J].Proceedings of the CSEE.2012 Vol.32 No.33

[9] Super Pond, noise and vibration of the motor[M] Beijing: Mechanical industry Press, 1982.

[10] Yunmou Tang. electromagnetic field Inside the motor[M] Beijing:Science Press, 1998. 